\title{
Repetitive Control based on Integral Sliding Mode Control of Matched Uncertain Systems
}

\author{
Nizar TOUJENI ${ }^{1}$, Chaouki MNASRI ${ }^{2}$, Moncef GASMI ${ }^{3}$ \\ Computer Laboratory for Industrial Systems (LISI) \\ National Institute of Applied Sciences and Technology (INSAT) \\ Carthage University, Tunisia
}

\begin{abstract}
This paper proposed an integral sliding mode control scheme based on repetitive control for uncertain repetitive processes with the presence of matched uncertainties, external disturbances and norm-bounded nonlinearities. A new method based on the combination of repetitive control and sliding mode approach is studied in order to use the robustness sensibility property of the sliding mode control to matched uncertainties and disturbances and to cancel gradually tracking error for periodic processes. A sufficient condition of the existence of sliding mode is studied based on basic repetitive control and a sliding mode controller is synthesized through linear matrix inequalities, which guarantees the stability along the periods of the controlled closed-loop process and the reachability of the sliding surface is ensured. Then, an adaptive integral sliding mode controller is synthesized to improve performances of the proposed control scheme. The effectiveness of the proposed controlled design schemes is proved by the use of a third order uncertain mechanical system and the simulation results using the new approaches give good performances.
\end{abstract}

Keywords-Repetitive control; 2D systems; matched uncertainties; integral sliding mode control; sliding surface; linear matrix inequality; reachability

\section{INTRODUCTION}

Repetitive Control (RC) has been applied to many engineering applications, such as robot manipulators, rotary systems, power supply systems, computer disk drives, etc. [1][4]. Based on the error signals in previous periods, the basic idea of $\mathrm{RC}$ is to improve transient responses on each pass by refining the control inputs in tracking problems for periodic operated dynamic systems. The basic RC is related to learning control [5]-[6] and it is formed of two main parts in order to produce a zero tracking error; a periodic signal is generated by the internal model originated from the idea offered by Wonham and Francis [7] and a proper compensator consists to stabilize the closed-loop feedback system.

Recently, an interesting theme in RC research fields is robust repetitive control design against system uncertainties. In practice, there exist a complex relationship between model and real system. When the controller is applied to real systems, disturbances and uncertainties must be absolutely considered and examined. Thus, they cause instability in the control system [8].

In other hand, many strategies using $\mathrm{RC}$ have been developed in order to solve this problem. Authors in [9]-[14] offer some methods of repetitive control system design for a class of linear system. They are based on two-dimensional (2D) continuous/discrete hybrid model. The traditional problem of repetitive controller design is reformed in an equivalent problem for a $2 \mathrm{D}$ continuous-discrete system and solved it by 2D Lyapunov theory by means linear matrix inequality (LMI) approach.

Therefore, classical RC has been associated and integrated with many robust control techniques [15]-[21] such as backstepping control, adaptive robust control, $H_{\infty}$ control, and sliding mode control (SMC). Referring to offered [8], [22], SMC has been looked as a good robust technique, especially for its insensibility to uncertainties satisfying the matching condition. Besides, SMC can offer good transient performance, fast response, and order reduction. These advantages make SMC a very practical and effective way in robust control design. Therefore, robustness against uncertainties matched to the control system can be only reached after the apparition of the sliding mode so-called reaching phase [23]-[24]. To ensure robustness in the overall closed-loop system response and eliminate the reaching phase, Integral Sliding Mode Control (ISMC) was proposed in [25]. The main contribution of this work is to propose a new Repetitive Integral Sliding Mode Control (RISMC) law of matched uncertain linear repetitive processes with external disturbances in order to achieve a zero tracking error.

The rest of this paper is organized as follows. Section II presents major work related to this study. The problem of equivalence $2 \mathrm{D}$ system and repetitive control is formulated in Section III. The sliding mode process is analyzed in Section III. Section V presents the reachability analysis. Section VI gives an illustrative example, and Section VII concludes this paper.

\section{RELATED WORKS}

The authors in [26] proposes a sliding mode based repetitive control system for periodic reference tracking in order to reduce transient overshoot, output noise, and chattering. The design method is simple with less restriction in stability conditions. The research work on [27] focused on improving the tracking performance and robust performance for turntable system by using a repetitive control design based on integral sliding mode. In [28], a quasi-sliding mode control of differential linear repetitive processes with unknown input disturbance is proposed. 
The main advantage of this work is to design a repetitive controller based on integral sliding mode control of matched uncertain systems with external disturbances by exploiting two major properties; the first is the insensibility to uncertainties satisfying the matching condition and the second is the improving control system performance in a periodic manner by including the learning capacity.

\section{PROBLEM FORMULATION AND PRELIMINARIES}

\section{A. Problem Statement}

Consider this uncertain system defined by:

$$
\left\{\begin{array}{l}
\dot{x}(t)=(A+\Delta A) x(t)+(B+\Delta B) u(t)+f(x, t)+\varpi(t) \\
y(t)=C x(t)
\end{array}\right.
$$

Where $x \in \mathbb{R}^{n}, y \in \mathbb{R}^{p}, u \in \mathbb{R}^{m}, f(x, t)$ and $\varpi(t) \in \mathbb{R}^{m}$ are the state vector, the output of the system, the input control, the vector of unmodelled dynamics and nonlinearities, and the vector of external disturbances respectively. Thus, $A$ is the state matrix, $B$ is the input matrix and $C$ is the output matrix with appropriate dimensions. $\Delta A \in \mathbb{R}^{n \times n}$ and $\Delta B \in \mathbb{R}^{n \times m}$ represent the system and the input matrix uncertainties. To complete the description of the uncertain dynamical system, the following assumptions are used:

\section{Assumption 1:}

(i) The pair $(A, B)$ is stabilizable and the input matrix $B$ has full rank.

(ii) $\Delta A, \Delta B, f(x, t)$ and $\varpi(t)$ are continuous on their arguments and they are unknown but have a known upper bound for all $(x, t) \in \mathbb{R}^{n} \times \mathbb{R}$.

(iii) Matching conditions: there exist functions $\Delta A_{m}, \Delta B_{m}$, $v(t)$ and $g(x, t)$, for all $(x, t) \in \mathbb{R}^{n} \times \mathbb{R}$, such that

$$
\begin{aligned}
& \Delta A=B \Delta A_{m} \\
& \Delta B=B \Delta B_{m} \\
& f(x, t)=B g(x, t) \\
& \varpi(t)=B v(t)
\end{aligned}
$$

Assumption 2: There exist known positive constants $a_{m}$, $b_{m}, \alpha_{m}$ and $\beta_{m}$ such that

$$
\begin{aligned}
& \left\|\Delta A_{m}\right\| \leq a_{m} \\
& \left\|\Delta B_{m}\right\| \leq b_{m}<1 \\
& \|f(x, t)\| \leq \alpha_{m}\|x\| \\
& \|\varpi(t)\| \leq \beta_{m}
\end{aligned}
$$

Where $\|\cdot\|$ denotes a signal quadrically-norm.

The tracking error between the periodic reference input and the output is defined by $e(t)=r(t)-y(t)$ where $r(t+T)=r(t)$ and $T$ is the fundamental repetition period. Consider the following function which is describe by $\xi(t)=\xi(k T+\tau):=\xi_{k}(\tau)$
$\Delta \xi(t)=\xi(t)-\xi(t-T):=\Delta \xi_{k}(\tau)$

Where $k \in \mathbb{N}$ and $0 \leq \tau<T$ are two independent parameters. The first describes learning between successive periods and the second characterizes control inside a period.

By applying function which defined in (4), plant model can be transformed in the following state-space equation

$$
\left\{\begin{array}{c}
\Delta \dot{x}_{k+1}(\tau)=(A+\Delta A) \Delta x_{k+1}(\tau)+(B+\Delta B) \Delta u_{k+1}(\tau) \\
+\Delta f_{k+1}\left(x_{k+1}, \tau\right)+\Delta \varpi_{k+1}(\tau) \\
e_{k+1}(\tau)=-C \Delta x_{k+1}(\tau)+e_{k}(\tau)
\end{array}\right.
$$

Let $\quad \eta_{k+1}(t)=\Delta x_{k+1}(\tau) \quad, \quad \tilde{\varpi}_{k+1}(t)=\Delta \varpi_{k+1}(\tau) \quad$, $\tilde{u}_{k+1}(t)=\Delta u_{k+1}(\tau)$, and $\tilde{f}_{k+1}\left(\eta_{k+1}, t\right)=\Delta f_{k+1}\left(x_{k+1}, \tau\right)$. Finally, the system (5) can be written as follows

$$
\left\{\begin{array}{c}
\dot{\eta}_{k+1}(t)=(A+\Delta A) \eta_{k+1}(t)+(B+\Delta B) \tilde{u}_{k+1}(t) \\
\quad+\tilde{f}_{k+1}\left(\eta_{k+1}, t\right)+\tilde{\varpi}_{k+1}(t) \\
e_{k+1}(t)=-C \eta_{k+1}(t)+e_{k}(t)
\end{array}\right.
$$

However, the result system (6) creates a 2D continuousdiscrete hybrid model of the repetitive control system within the presence of matched uncertainties in both state and input matrices and external disturbances.

Next, the main objective of this work is to make a sequence of control input functions in order to improve gradually the desired performance with the successive periods. The convergence condition of the tracking error and control input can be described as follows:

$$
\lim _{k \rightarrow \infty}\left\|e_{k}(t)\right\|=0, \lim _{k \rightarrow \infty}\left\|\tilde{u}_{k+1}(t)-\tilde{u}_{\infty}(t)\right\|=0
$$

Where $\tilde{u}_{\infty}$ is called the learned control.

In general, there are two distinct stability concepts that exist in the stability theory of linear repetitive processes; Asymptotic stability and Stability along the pass. The first concept ensures the existence of a limit profile described by a classical linear system state space model (1D system), the second concept guarantees that the existing limit profile is stable during the successive pass dynamics (2D system) [29]. In order to achieve stability of the controlled system (6), the concept stability along the pass must use.

\section{B. Preliminaries}

In order to explain and achieve main results, the following preliminaries are essential.

Lemma 1. [29] (Schur complement): Let $E, F$ and $G$ be given matrices with appropriate dimensions, where $\mathrm{E}$ and $\mathrm{G}$ are positive definite symmetric matrices. Then, the following inequality

$F^{T} G F-E<0$

is equivalent to 
$\left[\begin{array}{cc}-E & F^{T} \\ F & -G^{-1}\end{array}\right]<0$ or $\left[\begin{array}{cc}-G^{-1} & F \\ F^{T} & -E\end{array}\right]<0$

Definition 1. [28]: Consider the following linear time invariant repetitive system

$\left\{\begin{array}{l}\dot{x}_{k+1}(t)=A x_{k+1}(t)+B y_{k}(t) \\ y_{k+1}(t)=C x_{k+1}(t)+D y_{k}(t)\end{array}\right.$

The system (8) is stable along the pass if and only if three conditions are satisfied:

(i) $\rho(D)<1$

(ii) $\operatorname{Re}(\rho(A))<0$

(iii) All eigenvalues of $G(s)=C(s I-A)^{-1} B$ with $s=j \omega$,

for all real frequencies $\omega \geq 0$, have modulus strictly less than unity.

where the pair $[\rho(A), \rho(D)]$ represents respectively the spectral radius of a matrices $A$ and $D$.

In order to guarantee stability along the pass for system (10), the following lemma based on 2D Lyapunov stability theory is given.

Lemma 2. [30]: For linear time invariant repetitive system in (10), we introduce the 2D Lyapunov function $V_{k}(t)$ as

$$
\left\{\begin{array}{l}
V_{k}(t) \triangleq V_{1, k}(t)+V_{2, k}(t) \\
V_{1, k}(t) \triangleq x_{k+1}^{T}(t) P x_{k+1}(t) \\
V_{2, k}(t) \triangleq y_{k}^{T}(t) Q y_{k}(t)
\end{array}\right.
$$

where $\mathrm{P}$ and $\mathrm{Q}$ represent two symmetric positive definite matrices to be found. Then, the associated 2D Lyapunov function increment $\Delta V_{k}(t)$ is given by

$$
\left\{\begin{array}{l}
\Delta V_{k}(t) \triangleq \dot{V}_{1, k}(t)+\Delta V_{2, k}(t) \\
\dot{V}_{1, k}(t) \triangleq \dot{x}_{k+1}^{T}(t) P x_{k+1}(t)+x_{k+1}^{T}(t) P \dot{x}_{k+1}(t) \\
\Delta V_{2, k}(t) \triangleq y_{k+1}^{T}(t) Q y_{k+1}(t)-y_{k}^{T}(t) Q y_{k}(t)
\end{array}\right.
$$

The linear repetitive process in (10) is stable along the pass if and only if there exist two symmetric positive definite matrices $\mathrm{P}$ and $\mathrm{Q}$ such that $\Delta V_{k}(t)<0$.

The main goal of this paper is to make an ISMC law for the uncertain repetitive system described in (1). Details of the design process will be formulated in the next sections.

\section{SLIDING Mode STABILITY}

In the sliding mode literature, the ISMC design is making by two stages. The first step consists on choosing the proper switching function surface for the 2D uncertain system (6). The second step is to design a suitable relay-type controller to guarantee the sliding motion asymptotically stable. Now, details of those steps will be presented.

\section{A. Sliding Surface Choice}

The first phase is to choose sliding surface which takes in consideration to eliminate the reaching phase. In this study, the switching surface is specified by the equation:

$S_{k+1}(t)=B^{+} \eta_{k+1}(t)+\sigma_{k+1}(t)$

Where $S_{k+1}(0)=0$ for any initial conditions, $B^{+} \equiv\left(B^{T} B\right)^{-1} B^{T}$, and the function $\sigma_{k+1}(t) \in \mathbb{R}^{m}$ represents the solution of the following equation:

$\left\{\begin{array}{l}\dot{\sigma}_{k+1}(t)=-B^{+} A \eta_{k+1}(t)-\tilde{u}_{0_{k+1}}(t) \\ \sigma_{k+1}(0)=-B^{+} \eta_{k+1}(0)\end{array}\right.$

In addition, $\tilde{u}_{0_{k+1}}(t)$ is a nominal control law which should be designed with repetitive control feedback to achieve desired nominal performance. Then, we assume that the system (6) is forced to reach the sliding surface at the initial time $t_{0}$. The intrinsic condition of an ideal sliding motion can be achieved as follows

$\dot{S}_{k+1}(t)=S_{k+1}(t)=0$ for all $t \geq t_{0}$

To analyze the sliding motion, consider the time derivative of (13) given by

$$
\begin{aligned}
\dot{S}_{k+1} & =B^{+} \dot{\eta}_{k+1}+\dot{\sigma}_{k+1} \\
& =B^{+}\left(\Delta A \eta_{k+1}+(B+\Delta B) \tilde{u}_{k+1}+\tilde{\varpi}_{k+1}+\tilde{f}_{k+1}-B \tilde{u}_{0_{k+1}}\right) \\
& =\Delta A_{m} \eta_{k+1}+\left(I_{m}+\Delta B_{m}\right) \tilde{u}_{k+1}+\tilde{v}_{k+1}+\tilde{g}_{k+1}-\tilde{u}_{0_{k+1}}
\end{aligned}
$$

The equivalent control $\tilde{u}_{e q_{k+1}}(t)$ with the time derivative $\dot{S}_{k+1}(t)=0$ along the state trajectories can be written as

$\tilde{u}_{e q_{k+1}}(t)=\left(I_{m}+\Delta B_{m}\right)^{-1}\left(\tilde{u}_{0_{k+1}}-\Delta A_{m} \eta_{k+1}-\tilde{v}_{k+1}-\tilde{g}_{k+1}\right)$

The equivalent control is the average value such that the input control must hold the sliding motion on the sliding surface.

Remark 1. In (17), the matrix $\left(I_{m}+\Delta B_{m}\right)$ must be nonsingular which is guaranteed by Assumption 2.

\section{B. Stability of the Sliding Motion}

To obtain the sliding mode, the equivalent control (17) is not the input control law that must be applied to the system (6). In order to get the sliding motion expression, the value of $\tilde{u}_{e q_{k+1}}(t)$ can be substituted from (17) into (6), yields

$$
\begin{aligned}
\dot{\eta}_{k+1}(t) & =(A+\Delta A) \eta_{k+1}+\tilde{\varpi}_{k+1}+\tilde{f}_{k+1} \\
+ & (B+\Delta B)\left(I_{m}+\Delta B_{m}\right)^{-1} \\
& \times\left(\tilde{u}_{0_{k+1}}-\Delta A_{m} \eta_{k+1}-\tilde{v}_{k+1}-\tilde{g}_{k+1}\right) \\
= & A \eta_{k+1}(t)+B \tilde{u}_{0_{k+1}}(t)
\end{aligned}
$$

We remark from (18) that the effect of the uncertainties, nonlinearities and external disturbances during the sliding 
mode is completely rejected. As a result, uncertain system (6) is reduced to

$$
\left\{\begin{array}{l}
\dot{\eta}_{k+1}(t)=A \eta_{k+1}(t)+B \tilde{u}_{0_{k+1}}(t) \\
e_{k+1}(t)=-C \eta_{k+1}(t)+e_{k}(t)
\end{array}\right.
$$

The resulting system (19) is insensitive to matched uncertainties. Then, to achieve desired nominal performance, $\tilde{u}_{0_{k+1}}(t)$ is a nominal control input that can be designed by repetitive control.

\section{ISMC design based Repetitive Control}

The structure of the basic repetitive control system is shown by Fig. 1 where $G$ is the plant model. The transfer function of a basic repetitive controller is

$C_{R}(s)=\frac{1}{1-e^{-s T}}$

where $T$ is the known fundamental period of the reference periodic signal and $\Phi$ defines the output signal of the repetitive controller given by the following expression:

$\Phi_{k+1}(t)= \begin{cases}e_{k}(t), & 0 \leq t<T \\ \Phi_{k}(t)+e_{k}(t), & t \geq T\end{cases}$

The repetitive control law proposed of the system is

$u_{0_{k+1}}(t)=K_{1} x_{k+1}(t)+K_{2} \Phi_{k+1}(t)$

where the pair $\left(K_{1}, K_{2}\right)$ gains matrices with appropriate dimensions will be determined. Thus, these gains matrices ensure the stability along the period of the closed-loop system.

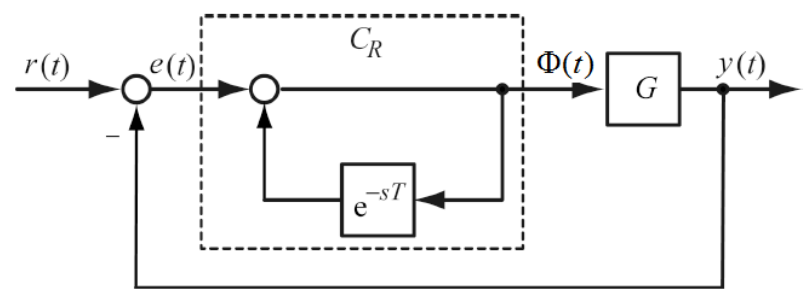

Fig. 1. Basic Repetitive Control System.

Next, the 2D nominal control input can be written as

$\tilde{u}_{0_{k+1}}(t)=K_{1} \eta_{k+1}(t)+K_{2} e_{k}(t)$

By replacing the expression of $\tilde{u}_{0_{k+1}}(t)$ into (19), the new 2D state space nominal dynamics can be written as

$$
\left\{\begin{array}{l}
\dot{\eta}_{k+1}(t)=\left(A+B K_{1}\right) \eta_{k+1}(t)+\left(B K_{2}\right) e_{k}(t) \\
e_{k+1}(t)=-C \eta_{k+1}(t)+e_{k}(t)
\end{array}\right.
$$

The next step consists to study the stability along the pass of the sliding mode process in (24) by designing gains controller $K_{1}$ and $K_{2}$. Therefore, to achieve the design process, the following theorem gives a sufficient condition for the stability along the pass of the sliding mode process in (24) by using the LMI method and the 2D system theory. After solving it, the designed sliding function in (13) is complete.

Theorem 1. The sliding mode process is stable along the pass if and only if there exist two symmetric positive definite matrices $X_{1}$ and $X_{2}$, and two matrices $W_{1}$ and $W_{2}$ such that the following LMI

$$
\left[\begin{array}{ccc}
A X_{1}+X_{1} A^{T}+B W_{1}+W_{1}^{T} B^{T} & (*) & (*) \\
W_{2}^{T} B^{T} & -X_{2} & (*) \\
C X_{1} & -X_{2} & -X_{2}
\end{array}\right]<0
$$

holds, then the closed-loop system (24) is stable along the pass, that is, the stabilization gains are given by

$\left\{\begin{array}{l}K_{1}=W_{1} \cdot X_{1}^{-1} \\ K_{2}=W_{2} \cdot X_{2}^{-1}\end{array}\right.$

Proof. Consider two symmetric positive-definite matrices $P$ and $Q$ and choose a candidate 2D Lyapunov function $V_{k}(t)$ by applying lemma 1 such that

$\left\{\begin{array}{l}V_{1, k}(t) \triangleq \eta_{k+1}^{T}(t) P \eta_{k+1}(t) \\ V_{2, k}(t) \triangleq e_{k}^{T}(t) Q e_{k}(t)\end{array}\right.$

Then, the associated 2D Lyapunov function increment $\Delta V_{k}(t)$ given by

$$
\left\{\begin{array}{l}
\dot{V}_{1, k}(t) \triangleq \dot{\eta}_{k+1}^{T}(t) P \eta_{k+1}(t)+\eta_{k+1}^{T}(t) P \dot{\eta}_{k+1}(t) \\
\Delta V_{2, k}(t) \triangleq e_{k+1}^{T}(t) Q e_{k+1}(t)-e_{k}^{T}(t) Q e_{k}(t)
\end{array}\right.
$$

Therefore, the Lyapunov function increment $\Delta V_{k}(t)$ can be transformed into

$$
\begin{aligned}
\Delta V_{k}(t)= & \dot{V}_{1, k}(t)+\Delta V_{2, k}(t) \\
= & \dot{\eta}_{k+1}^{T}(t) P \eta_{k+1}(t)+\eta_{k+1}^{T}(t) P \dot{\eta}_{k+1}(t) \\
& \quad+e_{k+1}^{T}(t) Q e_{k+1}(t)-e_{k}^{T}(t) Q e_{k}(t) \\
= & {\left[\left(A+B K_{1}\right) \eta_{k+1}(t)+\left(B K_{2}\right) e_{k}(t)\right]^{T} P \eta_{k+1}(t) } \\
& +\eta_{k+1}^{T}(t) P\left[\left(A+B K_{1}\right) \eta_{k+1}(t)+\left(B K_{2}\right) e_{k}(t)\right] \\
+ & {\left[-C \eta_{k+1}(t)+e_{k}(t)\right]^{T} Q\left[-C \eta_{k+1}(t)+e_{k}(t)\right] } \\
& \quad-e_{k}^{T}(t) Q e_{k}(t) \\
= & \varsigma^{T}(t) \psi \varsigma(t)
\end{aligned}
$$

where

$\left\{\begin{array}{l}\varsigma(t)=\left[\begin{array}{ll}\eta_{k+1}^{T}(t) & e_{k}^{T}(t)\end{array}\right]^{T} \\ \psi=\left[\begin{array}{cc}\left(A+B K_{1}\right)^{T} P+P\left(A+B K_{1}\right)+C^{T} Q C & (*) \\ \left(B K_{2}\right)^{T} P-Q C & 0\end{array}\right]\end{array}\right.$

Notice that (29) implies $\Delta V_{k}(t)<0$ (i.e. $\psi<0$ ) for any $\varsigma(t) \neq 0$. By Lemma 1 , stability along the pass of the sliding 
mode process in (24) is guaranteed. On the other hand, by Lemma 2 (Schur complement), LMI $\psi<0$ is equivalent to

$$
\left[\begin{array}{ccc}
\left(A+B K_{1}\right)^{T} P+P\left(A+B K_{1}\right) & (*) & (*) \\
\left(B K_{2}\right)^{T} P & -Q & (*) \\
Q C & -Q & -Q
\end{array}\right]<0
$$

After that, (31) pre-multiply and post-multiply by $\Sigma=\operatorname{diag}\left\{P^{-1}, Q^{-1}, Q^{-1}\right\}$ and its transpose respectively. Thus, LMI $\psi<0$ is finally equivalent to

$$
\left[\begin{array}{ccc}
P^{-1}\left(A+B K_{1}\right)^{T}+\left(A+B K_{1}\right) P^{-1} & (*) & (*) \\
Q^{-1}\left(B K_{2}\right)^{T} & -Q^{-1} & (*) \\
C P^{-1} & -Q^{-1} & -Q^{-1}
\end{array}\right]<0
$$

Let $X_{1}=P^{-1}, X_{2}=Q^{-1}, W_{1}=K_{1} X_{1}$, and $W_{2}=K_{2} X_{2}$. LMI (25) is obtained after replacing correspondent's terms in (32). Thus, the proof is finished.

\section{REACHABILITY ANALYSIS}

In the last section, a sufficient condition for the stability along the pass of the sliding mode process was derived. The next step consists to analyze the reachability of the sliding surface. Therefore, the reachability is a sufficient condition which to guarantee that the sliding mode process will converge to the sliding surface at each time instant.

\section{A. Repetitive Integral Sliding Mode Control Law}

In order to satisfy reachability condition, the following theorem proposes an RISMC law.

Theorem 2. Consider the $2 \mathrm{D}$ uncertain system (6) with the assumptions (1-2). Suppose that the sliding surface is given by (13) and $X_{1}, X_{2}, W_{1}$ and $W_{2}$ are solutions of the LMI (25). The RISMC is defined by

$\tilde{u}_{k+1}(t)=K_{1} \eta_{k+1}(t)+K_{2} e_{k}(t)-\rho \frac{S_{k+1}(t)}{\left\|S_{k+1}(t)\right\|}$

where

$$
\begin{gathered}
K_{1}=W_{1} \cdot X_{1}^{-1}, K_{2}=W_{2} \cdot X_{2}^{-1} \\
\rho=\frac{1}{1-b_{m}}\left[\varepsilon+\left(a_{m}+\alpha_{m}\left\|B^{+}\right\|+b_{m}\left\|K_{1}\right\|\right)\left\|\eta_{k+1}\right\|\right. \\
\left.\quad+b_{m}\left\|K_{2}\right\|\left\|e_{k}\right\|+\beta_{m}\left\|B^{+}\right\|\right]
\end{gathered}
$$

with $\varepsilon$ is a small positive scalar.

Proof. Let choose a Lyapunov function candidate to be

$$
V_{k+1}(t)=\frac{1}{2} S_{k+1}^{T}(t) \cdot S_{k+1}(t)
$$

In order to prove that the proposed RISMC law satisfies the reachability condition, substituting the value of (33) into (16) gives

$$
\begin{aligned}
\dot{S}_{k+1}= & \Delta A_{m} \eta_{k+1}+\left(I_{m}+\Delta B_{m}\right) \tilde{u}_{k+1}+\tilde{v}_{k+1}+\tilde{g}_{k+1}-\tilde{u}_{0_{k+1}} \\
= & \left(\Delta A_{m}+\Delta B_{m} K_{1}\right) \eta_{k+1}+\Delta B_{m} K_{2} e_{k}+\tilde{v}_{k+1}+\tilde{g}_{k+1} \\
& -\rho\left(I_{m}+\Delta B_{m}\right) \frac{S_{k+1}}{\left\|S_{k+1}\right\|}
\end{aligned}
$$

Pre-multiplying both sides of (36) by $S_{k+1}^{T}$ yields

$$
\begin{array}{r}
S_{k+1}^{T} \dot{S}_{k+1}=S_{k+1}^{T}\left[\left(\Delta A_{m}+\Delta B_{m} K_{1}\right) \eta_{k+1}+\Delta B_{m} K_{2} e_{k}\right. \\
+\tilde{v}_{k+1}+\tilde{g}_{k+1}-\rho\left(I_{m}+\Delta B_{m}\right) \frac{S_{k+1}}{\left\|S_{k+1}\right\|} \\
=S_{k+1}^{T}\left[\left(\Delta A_{m}+\Delta B_{m} K_{1}\right) \eta_{k+1}+\Delta B_{m} K_{2} e_{k}\right. \\
\left.+\tilde{v}_{k+1}+\tilde{g}_{k+1}\right]-\rho\left(I_{m}+\Delta B_{m}\right) \frac{S_{k+1}^{T} S_{k+1}}{\left\|S_{k+1}\right\|}
\end{array}
$$

and by using the property $S_{k+1}^{T} S_{k+1}=\left\|S_{k+1}\right\|^{2}$, (37) becomes

$$
\begin{gathered}
\dot{V}_{k+1}=S_{k+1}^{T}\left[\left(\Delta A_{m}+\Delta B_{m} K_{1}\right) \eta_{k+1}+\Delta B_{m} K_{2} e_{k}\right. \\
\left.\quad+\tilde{v}_{k+1}+\tilde{g}_{k+1}\right]-\rho\left(I_{m}+\Delta B_{m}\right)\left\|S_{k+1}\right\| \\
=S_{k+1}^{T}\left[\left(\Delta A_{m}+\Delta B_{m} K_{1}\right) \eta_{k+1}+\Delta B_{m} K_{2} e_{k}\right. \\
\left.\quad+\tilde{v}_{k+1}+\tilde{g}_{k+1}\right]-\rho\left\|S_{k+1}\right\|-\rho \Delta B_{m}\left\|S_{k+1}\right\| \\
\leq S_{k+1}^{T}\left[\left(\Delta A_{m}+\Delta B_{m} K_{1}\right) \eta_{k+1}+\Delta B_{m} K_{2} e_{k}\right. \\
\left.\quad+\tilde{v}_{k+1}+\tilde{g}_{k+1}\right]-\rho\left(1-b_{m}\right)\left\|S_{k+1}\right\| \\
\leq\left\|S_{k+1}\right\|\left[\left(\left\|\Delta A_{m}\right\|+\left\|\Delta B_{m} K_{1}\right\|\right)\left\|\eta_{k+1}\right\|+\left\|\Delta B_{m} K_{2}\right\|\left\|e_{k}\right\|\right. \\
\left.\quad+\left\|\tilde{v}_{k+1}\right\|+\left\|\tilde{g}_{k+1}\right\|\right]-\rho\left(1-b_{m}\right)\left\|S_{k+1}\right\|
\end{gathered}
$$

Finally, by using Assumption 2, (38) can be written as

$$
\begin{array}{r}
\dot{V}_{k+1} \leq\left\|S_{k+1}\right\|\left[\left(a_{m}+\alpha_{m}\left\|B^{+}\right\|+b_{m}\left\|K_{1}\right\|\right)\left\|\eta_{k+1}\right\|\right. \\
\left.+b_{m}\left\|K_{2}\right\|\left\|e_{k}\right\|+\beta_{m}\left\|B^{+}\right\|-\rho\left(1-b_{m}\right)\right]
\end{array}
$$

The reachability condition is guaranteed if and only if $\dot{V}_{k+1}<0$ is satisfied. Then, in order to enforce it, any choice of $\rho$ must satisfy the following condition

$$
\begin{gathered}
\rho>\frac{1}{1-b_{m}}\left[\left(a_{m}+\alpha_{m}\left\|B^{+}\right\|+b_{m}\left\|K_{1}\right\|\right)\left\|\eta_{k+1}\right\|\right. \\
\left.+b_{m}\left\|K_{2}\right\|\left\|e_{k}\right\|+\beta_{m}\left\|B^{+}\right\|\right]
\end{gathered}
$$

Let $\varepsilon$ is a small positive scalar, the inequality in (39) becomes

$$
S_{k+1}^{T}(t) \dot{S}_{k+1}(t) \leq-\varepsilon\left\|S_{k+1}(t)\right\|<0
$$

Besides, the initial value of $S_{k+1}(t)$ is provided by $S_{k+1}(0)=0$ for any initial conditions. So, the reachability of the sliding surface is guaranteed. That ended the proof. 


\section{B. Adaptative Repetitive Integral Sliding Mode Control Law}

Applicability of the designed RISMC law presents two major problems. The first is the chattering phenomenon and the second is the difficulty to get the exact upper bound values of uncertainties and external disturbances [31].

In order to overcome these problems, we will present an Adaptive RISMC (ARISMC) law in this section. To get there, we start by rewriting (6) as

$$
\begin{aligned}
\dot{\eta}_{k+1}=(A+\Delta A) \eta_{k+1} & +(B+\Delta B) \tilde{u}_{k+1} \\
& +B \tilde{v}_{k+1}+B \tilde{g}_{k+1} \\
=A \eta_{k+1}+(B+ & \Delta B) \tilde{u}_{k+1} \\
& +B\left(\Delta A_{m} \eta_{k+1}+\tilde{v}_{k+1}+\tilde{g}_{k+1}\right)
\end{aligned}
$$

Let $\mu_{k+1}=\Delta A_{m} \eta_{k+1}+\tilde{v}_{k+1}+\tilde{g}_{k+1}$, (42) becomes

$\left\{\begin{array}{l}\dot{\eta}_{k+1}=A \eta_{k+1}+(B+\Delta B) \tilde{u}_{k+1}+B \mu_{k+1} \\ e_{k+1}=-C \eta_{k+1}+e_{k}\end{array}\right.$

To complete the description of the $2 \mathrm{D}$ uncertain system (43), the following assumption is used:

Assumption 3: There are unknown positive constants $\delta_{1}$ and $\delta_{2}$, for all $(x, t) \in \mathbb{R}^{n} \times \mathbb{R}$, such that

$\left\|\mu_{k+1}\right\| \leq \delta_{1}\left\|\eta_{k+1}\right\|+\delta_{2}$

To achieve design of ARISMC law, two steps are necessaries. First step, simple adaptation laws will be proposed for the upper bound of $\left\|\mu_{k+1}\right\|$. Second step consists to design a control law using this result, adaptive upper bound [31]. Let consider $\bar{\delta}_{1}$ and $\bar{\delta}_{2}$, respectively, the adaptive parameters about $\delta_{1}$ and $\delta_{2}$. The proposed adaptive upper bound of $\left\|\mu_{k+1}\right\|$ is defined by

$\bar{\mu}_{k+1}=\bar{\delta}_{1}\left\|\eta_{k+1}\right\|+\bar{\delta}_{2}$

Now, define the parameter adaptation errors as $\tilde{\delta}_{1}=\bar{\delta}_{1}-\delta_{1}$ and $\tilde{\delta}_{2}=\bar{\delta}_{2}-\delta_{2}$. The simple adaptation laws proposed for the upper bound of $\left\|\mu_{k+1}\right\|$ is

$$
\begin{aligned}
& \dot{\tilde{\delta}}_{1} \triangleq \phi_{1}\left\|\eta_{k+1}\right\|\left\|S_{k+1}\right\| \\
& \dot{\tilde{\delta}}_{2} \triangleq \phi_{2}\left\|S_{k+1}\right\|
\end{aligned}
$$

where $\phi_{1}$ and $\phi_{2}$ are positive adaptation gains. However, $\delta_{1}$ and $\delta_{2}$ are assumed as constant values. Then, the result adaptation laws can be rewritten as

$$
\begin{aligned}
& \dot{\bar{\delta}}_{1} \triangleq \phi_{1}\left\|\eta_{k+1}\right\|\left\|S_{k+1}\right\| \\
& \dot{\bar{\delta}}_{2} \triangleq \phi_{2}\left\|S_{k+1}\right\|
\end{aligned}
$$

After that, by integrating (47), the adaptive parameters are described by the following expressions:

$$
\begin{aligned}
& \bar{\delta}_{1}=\bar{\delta}_{1 i}+\phi_{1} \int_{t_{0}+(k+1) T}^{t+(k+1) T}\left\|\eta_{k+1}\right\|\left\|S_{k+1}\right\| d t \\
& \bar{\delta}_{2}=\bar{\delta}_{2 i}+\phi_{2} \int_{t_{0}+(k+1) T}^{t+(k+1) T}\left\|S_{k+1}\right\| d t
\end{aligned}
$$

where $\bar{\delta}_{1 i}$ and $\bar{\delta}_{2 i}$ represents, respectively, the initial values of $\bar{\delta}_{1}$ and $\bar{\delta}_{2}$. Then, we can expose the following theorem.

Theorem 3. Consider the 2D uncertain system (6) with the assumptions (1-3). Suppose that the sliding surface is given by (13) and $X_{1}, X_{2}, W_{1}$ and $W_{2}$ are solutions of the LMI (25). The ARISMC is defined by

$\tilde{u}_{k+1}(t)=K_{1} \eta_{k+1}(t)+K_{2} e_{k}(t)-\hat{\rho} \frac{S_{k+1}(t)}{\left\|S_{k+1}(t)\right\|}$

where

$K_{1}=W_{1} \cdot X_{1}^{-1}, K_{2}=W_{2} \cdot X_{2}^{-1}$

$\hat{\rho}_{1}=\varepsilon+\left(b_{m}\left\|K_{1}\right\|+\bar{\delta}_{1}\right)\left\|\eta_{k+1}\right\|+b_{m}\left\|K_{2}\right\|\left\|e_{k}\right\|+\bar{\delta}_{2}$

$\hat{\rho}=\frac{1}{1-b_{m}} \hat{\rho}_{1}$

with $\varepsilon$ is a small positive scalar. By employing the adaptation laws (46) and the control law (49), $S_{k+1}=0$ is stable along the pass.

Proof. Let choose an improved Lyapunov function candidate instead of $S_{k+1}^{T} \dot{S}_{k+1}$ to be

$$
V_{k+1}=\frac{1}{2}\left(S_{k+1}^{T} S_{k+1}+\phi_{1}^{-1} \tilde{\delta}_{1}^{2}+\phi_{2}^{-1} \tilde{\delta}_{2}^{2}\right)
$$

where $\phi_{1}$ and $\phi_{2}$ are defined in (46). Substituting the value of (49) into (16) gives

$$
\begin{aligned}
\dot{S}_{k+1}= & \Delta A_{m} \eta_{k+1}+\left(I_{m}+\Delta B_{m}\right) \tilde{u}_{k+1}+\tilde{v}_{k+1}+\tilde{g}_{k+1}-\tilde{u}_{0_{k+1}} \\
= & \mu_{k+1}-K_{1} \eta_{k+1}-K_{2} e_{k} \\
& +\left(I_{m}+\Delta B_{m}\right)\left(K_{1} \eta_{k+1}+K_{2} e_{k}-\frac{1}{1-b_{m}} \hat{\rho}_{1} \frac{S_{k+1}(t)}{\left\|S_{k+1}(t)\right\|}\right) \\
= & \mu_{k+1}+\Delta B_{m} K_{1} \eta_{k+1}+\Delta B_{m} K_{2} e_{k} \\
& -\hat{\rho}_{1} \frac{1}{1-b_{m}}\left(I_{m}+\Delta B_{m}\right) \frac{S_{k+1}}{\left\|S_{k+1}\right\|}
\end{aligned}
$$

Pre-multiplying both sides of (52) by $S_{k+1}^{T}$ yields

$$
\begin{array}{r}
S_{k+1}^{T} \dot{S}_{k+1}=S_{k+1}^{T}\left(\mu_{k+1}+\Delta B_{m} K_{1} \eta_{k+1}+\Delta B_{m} K_{2} e_{k}\right) \\
-\hat{\rho}_{1} \frac{1}{1-b_{m}}\left(I_{m}+\Delta B_{m}\right)\left\|S_{k+1}\right\|
\end{array}
$$

Then, the Lyapunov function increment is given by 


$$
\begin{aligned}
\dot{V}_{k+1}= & S_{k+1}^{T} \dot{S}_{k+1}+\phi_{1}^{-1} \tilde{\delta}_{1} \dot{\tilde{\delta}}_{1}+\phi_{2}^{-1} \tilde{\delta}_{2} \dot{\tilde{\delta}}_{2} \\
= & S_{k+1}^{T}\left(\mu_{k+1}+\Delta B_{m} K_{1} \eta_{k+1}+\Delta B_{m} K_{2} e_{k}\right) \\
& -\hat{\rho}_{1} \frac{1}{1-b_{m}}\left(I_{m}+\Delta B_{m}\right)\left\|S_{k+1}\right\|+\phi_{1}^{-1} \tilde{\delta}_{1} \dot{\tilde{\delta}}_{1}+\phi_{2}^{-1} \tilde{\delta}_{2} \dot{\tilde{\delta}}_{2} \\
= & S_{k+1}^{T}\left(\mu_{k+1}+\Delta B_{m} K_{1} \eta_{k+1}+\Delta B_{m} K_{2} e_{k}\right) \\
& -\hat{\rho}_{1} \frac{1}{1-b_{m}}\left(I_{m}+\Delta B_{m}\right)\left\|S_{k+1}\right\| \\
& +\tilde{\delta}_{1}\left\|\eta_{k+1}\right\|\left\|S_{k+1}\right\|+\tilde{\delta}_{2}\left\|S_{k+1}\right\| \\
= & S_{k+1}^{T}\left(\mu_{k+1}+\Delta B_{m} K_{1} \eta_{k+1}+\Delta B_{m} K_{2} e_{k}\right) \\
& -\hat{\rho}_{1} \frac{1}{1-b_{m}}\left(I_{m}+\Delta B_{m}\right)\left\|S_{k+1}\right\| \\
& +\left(\bar{\delta}_{1}-\delta_{1}\right)\left\|\eta_{k+1}\right\|\left\|S_{k+1}\right\|+\left(\bar{\delta}_{2}-\delta_{2}\right)\left\|S_{k+1}\right\|
\end{aligned}
$$

Then, by using Assumption 2, (54) can be written as

$$
\begin{aligned}
\dot{V}_{k+1} \leq & \left(\left\|\mu_{k+1}\right\|+b_{m}\left\|K_{1}\right\|\left\|\eta_{k+1}\right\|+b_{m}\left\|K_{2}\right\|\left\|e_{k}\right\|\right. \\
& \left.+\frac{b_{m}}{1-b_{m}} \hat{\rho}_{1}\right)\left\|S_{k+1}\right\|-\frac{1}{1-b_{m}} \hat{\rho}_{1}\left\|S_{k+1}\right\| \\
& +\left[\left(\bar{\delta}_{1}\left\|\eta_{k+1}\right\|+\bar{\delta}_{2}\right)-\left(\delta_{1}\left\|\eta_{k+1}\right\|+\delta_{2}\right)\right]\left\|S_{k+1}\right\| \\
\leq & {\left[\left\|\mu_{k+1}\right\|-\left(\delta_{1}\left\|\eta_{k+1}\right\|+\delta_{2}\right)\right]\left\|S_{k+1}\right\| } \\
+ & \left(b_{m}\left\|K_{1}\right\|\left\|\eta_{k+1}\right\|+b_{m}\left\|K_{2}\right\|\left\|e_{k}\right\|\right. \\
& \left.+\bar{\delta}_{1}\left\|\eta_{k+1}\right\|+\bar{\delta}_{2}-\hat{\rho}_{1}\right)\left\|S_{k+1}\right\|
\end{aligned}
$$

The reachability condition is guaranteed if and only if $\dot{V}_{k+1}<0$ is satisfied. Finally, by using Assumption 3, it is sufficient to choose the value of $\hat{\rho}_{1}$ as

$\hat{\rho}_{1}>b_{m}\left\|K_{1}\right\|\left\|\eta_{k+1}\right\|+b_{m}\left\|K_{2}\right\|\left\|e_{k}\right\|+\bar{\delta}_{1}\left\|\eta_{k+1}\right\|+\bar{\delta}_{2}$

Let $\varepsilon$ is a small positive scalar, the inequality in (55) becomes

$$
\dot{V}_{k+1} \leq-\varepsilon\left\|S_{k+1}(t)\right\|<0
$$

In addition, the initial value of $S_{k+1}(t)$ is provided by $S_{k+1}(0)=0$ for any initial conditions. So, the reachability of the sliding surface is guaranteed. That ended the proof.

Remark 2. In this proposition, the rate of parameter adaptation is adjusted by an appropriate choose of $\left\{\bar{\delta}_{1 i}, \bar{\delta}_{2 i}\right\}$ and $\left\{\phi_{1}, \phi_{2}\right\}$. In practice, choices of adaptation gains are limited by many practical considerations such as the bound of control input and other parameters.

\section{ILlustrative EXAMPLE}

In order to prove the validity and the effectiveness of the new proposed controlled design schemes, consider the following nominal model of a submarine from [32].

$$
\left\{\begin{array}{l}
\dot{x}(t)=\left[\begin{array}{ccc}
0 & 1 & 0 \\
-0.0071 & -0.111 & 0.12 \\
0 & 0.07 & -0.3
\end{array}\right] x(t)+\left[\begin{array}{c}
0 \\
-0.095 \\
0.072
\end{array}\right] u(t) \\
y(t)=\left[\begin{array}{lll}
0 & 0 & 1
\end{array}\right] x(t)
\end{array}\right.
$$

where the state vector is defined by $x(t)=\left[\begin{array}{lll}\theta & \frac{d \theta}{d t} & \alpha\end{array}\right]^{T}$ with $\theta$ is the inclination of the submarine and $\alpha$ is the angle of attack. It's assumed that can only measure $\frac{d \theta}{d t}$ and suppose that

$$
\begin{aligned}
& \Delta A=\left[\begin{array}{ccc}
0 & -\zeta_{1} & 0 \\
0.0071 \zeta_{2} & 0.111 \zeta_{2} & -0.12 \zeta_{2} \\
0 & -0.07 \zeta_{3} & 0.3 \zeta_{3}
\end{array}\right] \\
& \Delta B=\left[\begin{array}{c}
0 \\
0.095 \zeta_{2} \\
-0.072 \zeta_{3}
\end{array}\right], \zeta_{i}=\frac{\lambda_{i}}{1+\lambda_{i}} \text { for } i=1, \ldots, 3 \\
& \varpi(t)=\left[\begin{array}{c}
0 \\
-0.02 \sin \left(\frac{3 \pi}{4 T} t+\frac{T}{4}\right) \\
0.02 \sin \left(\frac{3 \pi}{4 T} t+\frac{T}{4}\right)
\end{array}\right], f(x, t)=\left[\begin{array}{l}
0 \\
0 \\
0
\end{array}\right]
\end{aligned}
$$

Consider the following periodic reference input:

$$
r(t)=0.5 \sin \left(\frac{2 \pi}{T} t\right)+0.5 \sin \left(\frac{4 \pi}{T} t\right)
$$

Therefore, a feasible solution of the LMI (25) is defined as

$$
\begin{aligned}
& X_{1}=\left[\begin{array}{ccc}
64.9274 & -0.0000 & -1.6712 \\
-0.0000 & 26.0324 & -19.7298 \\
-1.6712 & -19.7298 & 98.3993
\end{array}\right], X_{2}=72.1792 \\
& W_{1}=\left[\begin{array}{lll}
267.0619 & 276.3066 & 112.2730
\end{array}\right], W_{2}=-274.0254 \\
& \begin{cases}K_{1}=\left[\begin{array}{lll}
4.2146 & 13.5996 & 3.9394
\end{array}\right],\left\|K_{1}\right\|=14.7726 \\
K_{2}=-3.7965, & \left\|K_{2}\right\|=3.7965\end{cases}
\end{aligned}
$$

For $\lambda_{i}=0.1, i=1, \ldots, 3$, we get

$$
\begin{gathered}
\Delta A_{m}=\left[\begin{array}{lll}
-0.0047 & -0.1097 & 0.2322
\end{array}\right],\left\|\Delta A_{m}\right\|=0.2569 \\
\Delta B_{m}=-0.1000,\left\|\Delta B_{m}\right\|=0.1000,\left\|B^{+}\right\|=8.3892 \\
\|\varpi(t)\|=0.0283\left|\sin \left(\frac{3 \pi}{4 T} t+\frac{T}{4}\right)\right|
\end{gathered}
$$


Then, using assumption 1, we get $a_{m}=0.3569$, $b_{m}=0.2000, \alpha_{m}=0.0010, \beta_{m}=0.0283$ and $\varepsilon=0.1000$. In addition, using (48), let $\bar{\delta}_{1 i}=0.1, \phi_{1}=0.1, \bar{\delta}_{2 i}=0.1$ and $\phi_{2}=0.1$.

The simulation results are given for the fundamental repetition period $T=10 \mathrm{~s}$ and the initial state vector $x_{1}(0)=\left[\begin{array}{lll}0.1 & 0.2 & 0.3\end{array}\right]^{T}$.

Figures 2 and 3 show the evolution of modulation gain $\rho$, switching function $S(t)$, reference $r(t)$ and output $y(t)$, tracking error $e(t)$, and controller $\tilde{u}(t)$ using the proposed RISMC (33-34) and ARISMC (49-50), respectively.

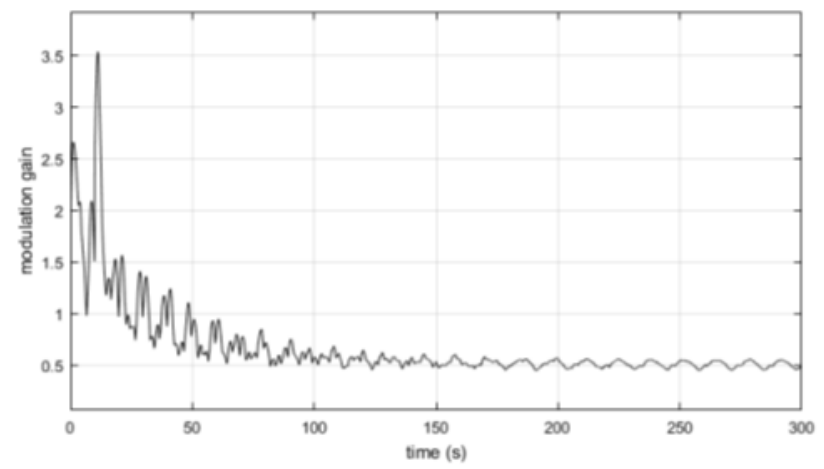

(a)

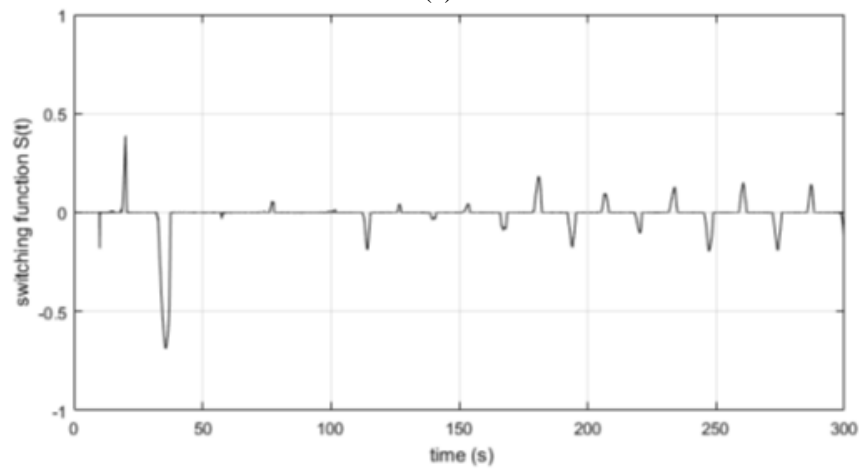

(b)

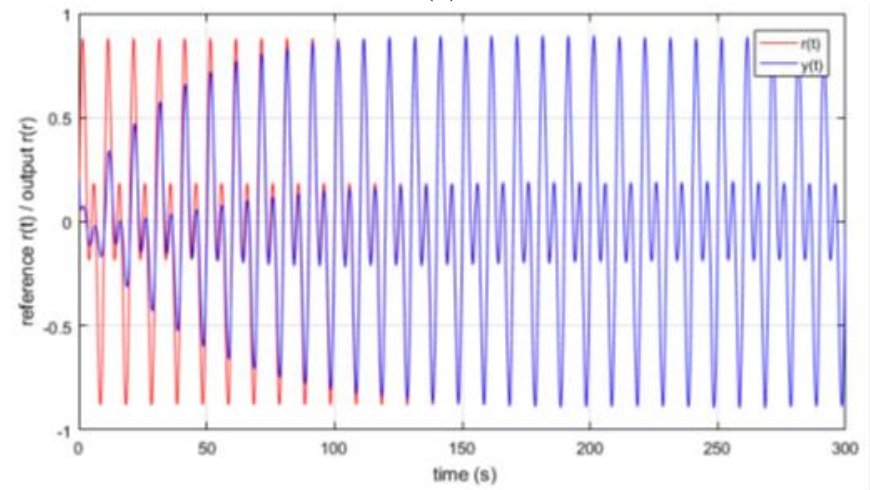

(c)

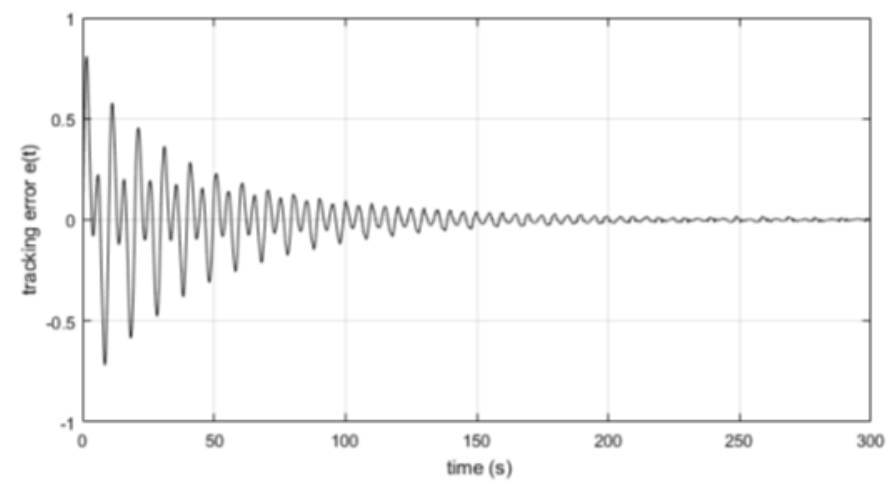

(d)

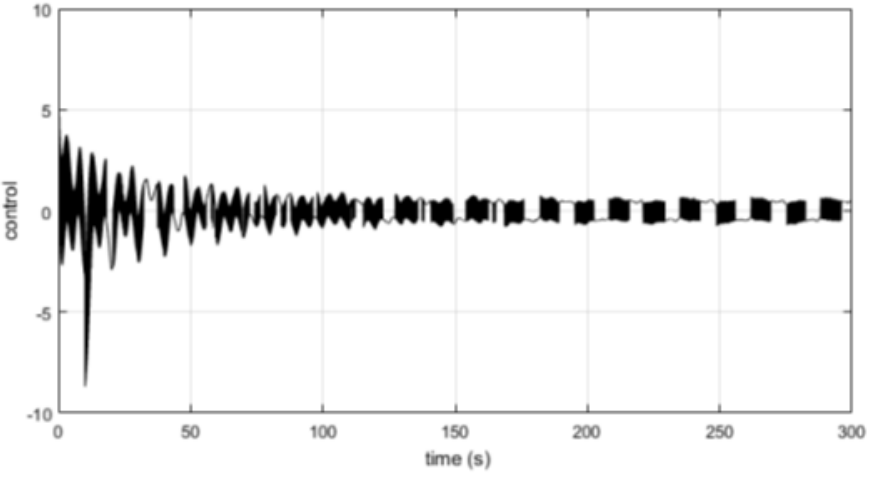

(e)

Fig. 2. Simulation Results using RISMC: Modulation Gain $\rho$ (a), Switching Function $S(t)$ (b), Reference $r(t)$ and Output $y(t)$ (c), Tracking Error $e(t)(\mathrm{d})$, Controller $\tilde{u}(t)(\mathrm{e})$.

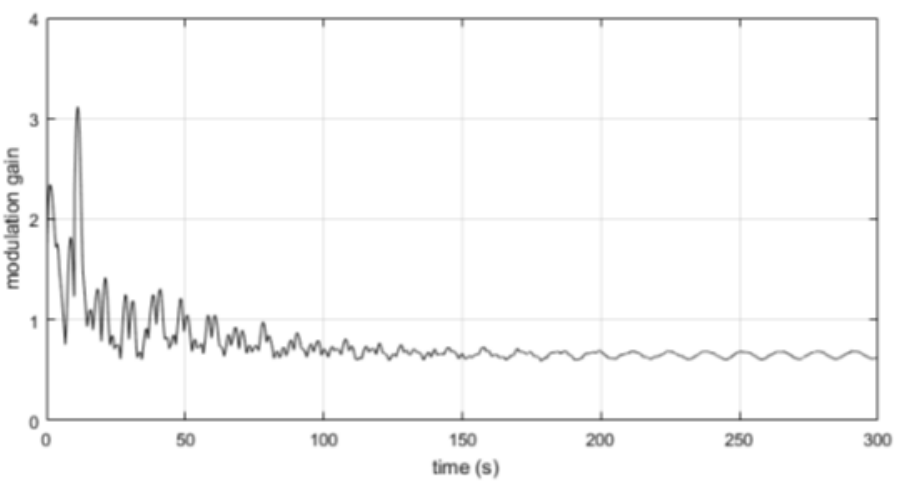

(a)

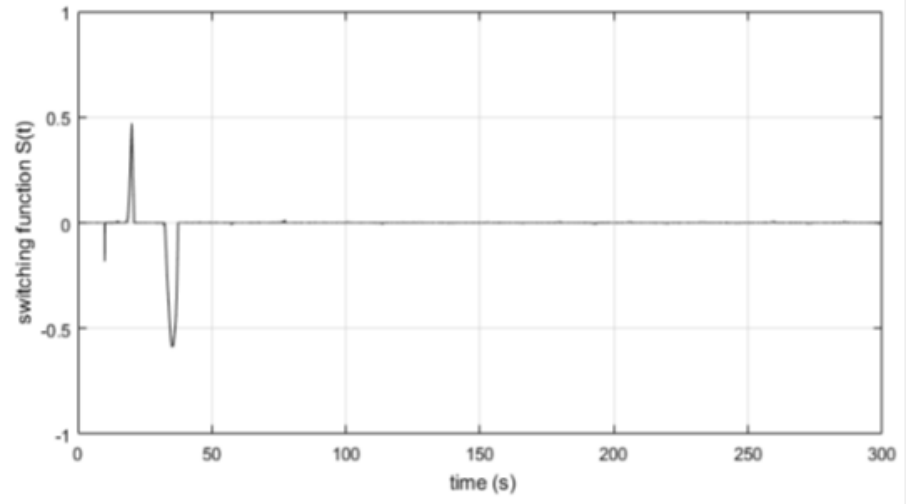

(b) 


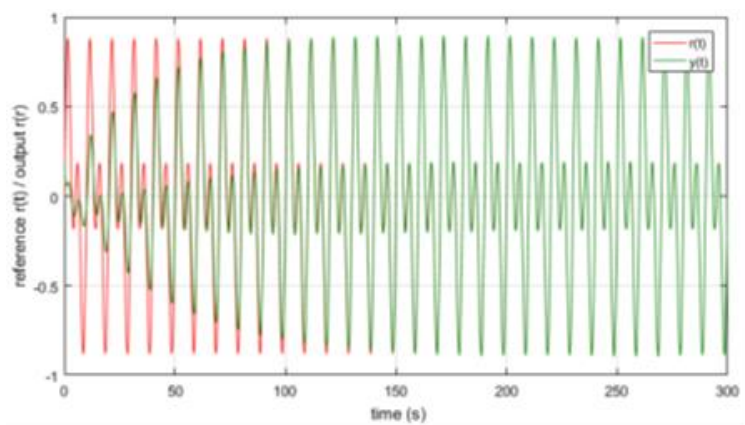

(c)

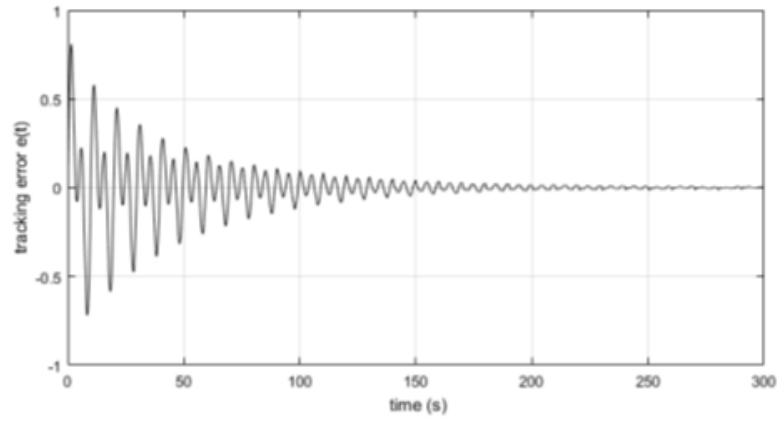

(d)

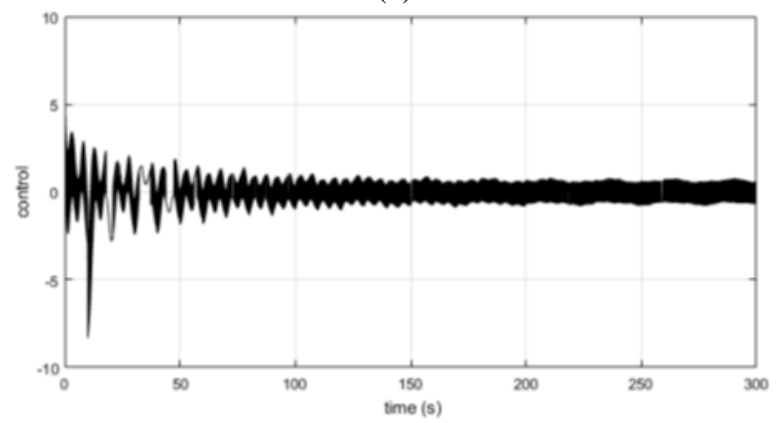

(e)

Fig. 3. Simulation results using ARISMC: modulation gain $\rho$ (a), switching function $S(t)$ (b), reference $r(t)$ and output $y(t)$ (c), tracking error $e(t)$ (d), controller $\tilde{u}(t)$ (e).

Thus, it is easy to remark that the proposed control laws ensure the stability along the pass of the closed-loop system. However, the chattering phenomenon appears in the evolution of the controller for the RISMC approach. So, the ARISMC approach has overcome this problem and the controller evolution confirms the elimination of discontinuities in high frequency.

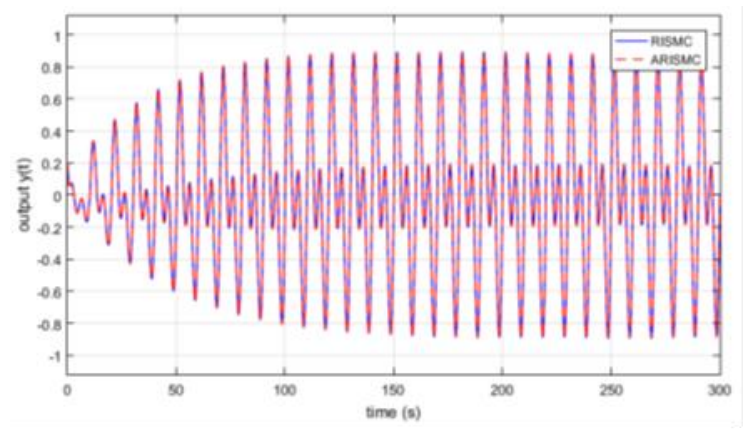

Fig. 4. Output Evolution According to RISMC and ARISMC.

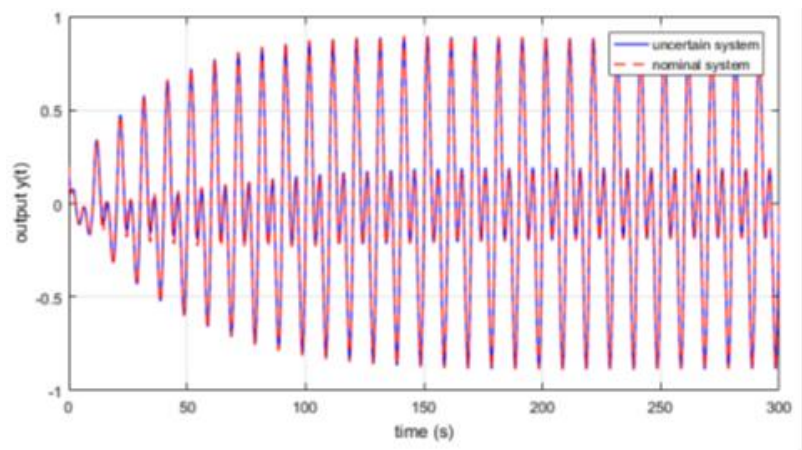

Fig. 5. Output Evolution According to ARISMC for the Uncertain and Nominal System.

Figure 4 shows the evolution of the output $y(t)$ for both RISMC and ARISMC methods, and the evolution of $y(t)$ for both uncertain and nominal systems are presented in Fig. 5. It is obvious from Fig. 4 that the ARISMC approach almost gives the same performances as the RISMC approach. In addition, evolution outputs from the uncertain and nominal systems are superposed (see Fig. 5). Then, simulation results confirm the robustness of the proposed methods.

\section{CONCLUSION}

In this paper, the problem of integral sliding mode control of uncertain repetitive processes in the presence of matched uncertainties, external disturbances and norm-bounded nonlinearities was studied.

A combination of repetitive control and sliding mode approach was exploited to reject the effect of uncertainties, nonlinearities and disturbances and a sufficient condition of the existence of sliding mode was studied based on basic repetitive control and a sliding mode controller which was synthesized by means of linear matrix inequalities, that guarantees the stability along the periods of the controlled closed-loop process. An adaptive integral sliding mode controller based on repetitive control was also proposed to improve performances of the synthesized control scheme.

The simulation results using the new approaches have given good performances and confirm the efficiency of the new proposed controlled design schemes. In addition, the RISMC problem for linear repetitive processes with mismatched uncertainties can be examined as our future work.

\section{REFERENCES}

[1] L. Zhou, J. She, C. Li, and C. Pan, "Robust aperiodic-disturbance rejection in an uncertain modified repetitive-control system," International Journal of Applied Mathematics and Computer Science, vol. 26, no. 2, pp. 285-295, 2016.

[2] S. G. Yuan, M. Wu, B. G. Xu, and R. J. Liu, "Design of Discrete-time Repetitive Control System Based on Two-dimensional Model," International Journal of Automation and Computing, vol. 9, no. 2, pp. 165-170, 2012.

[3] Z. Li, W. Zhang, Y. Zhang, and X. Xu, "Robust Repetitive Control Design and Its Application on Linear Servo Systems," International Journal of Precision Engineering and Manufacturing, vol. 16, no. 1, pp. 21-29, 2015.

[4] J. D. Ratcliffe, J. J. Hatonen, P. L. Lewin, E. Rogers, and D. H. Owens, "Repetitive control of synchronized operations for process applications," 
International Journal of Adaptive Control and Signal Processing, vol. 21, pp. 300-325, 2007.

[5] Y. Song, X. Yu, G. Chen, J. X. Xu, and Y. P. Tian, "Time delayed repetitive learning control for chaotic systems," International Journal of Bifurcation and Chaos, vol. 12, no. 5, pp. 1057-1065, 2002.

[6] J. X. Xu and R. Yan, "On repetitive learning control for periodic tracking tasks," IEEE Transactions on Automatic Control, vol. 51, no. 11, pp. 1842-1848, 2006.

[7] B. A. Francis and W. M. Wonham, "The internal model principle for linear multivariable regulators," Applied Mathematics and Optimization, vol. 2, pp. 170-194, 1975.

[8] C. Mnasri and M. Gasmi, "LMI-based adaptative fuzzy integral sliding mode control of mismatched uncertain systems," International Journal of Applied Mathematics and Computer Science, vol. 21, no. 4, pp. 605615, 2011.

[9] M. Wu, Y. H. Lan, J. She, Y. He, and L. Xu, "Optimal repetitive control based on two-dimensional model," International Journal of Innovative Computing, Information and Control, vol. 8, no. 3A, 2012.

[10] Z. H. Wang, L. Z. Yi, Y. H. Lan, and C. X. Chen, "Design of observerbased discrete repetitive-control system based on 2D model," Journal of Central South University of Technology, vol. 21, pp. 4236-4243, 2014.

[11] L. Zhou, J. She, and S. Zhou, "A 2D system approach to the design of a robust modified repetitive-control system with a dynamic outputfeedback controller," International Journal of Applied Mathematics and Computer Science, vol. 24, no. 2, pp. 325-334, 2014.

[12] M. A. Emelianov, P. V. Pakshin, K. Galkowski, and E. Rogers, "Stabilization of Differential Repetitive Processes," Automation and Remote Control, vol. 76, no. 5, pp. 786-800, 2015.

[13] P. Yu, M. Wu, J. She, and Q. Lei, "Robust repetitive control and disturbance rejection based on two-dimensional model and equivalentinput-disturbance approach," Asian Journal of Control, vol. 18, no. 6, pp. 1-11, 2016.

[14] L. Zhou and J. She, "Aperiodic Disturbance Rejection in a Modified Repetitive-control System," International Journal of Control, Automation and Systems, vol. 14, no. 4, pp. 883-892, 2016.

[15] Q. Zhu, J. X. Xu, S. Yang, and G. D. Hu, "Adaptive backstepping repetitive learning control design for nonlinear discrete-time systems with periodic uncertainties," International Journal of Adaptive Control and Signal Processing, vol. 29, pp. 524-535, 2015.

[16] Y. H. Yang and C. L. Chen, "Spatial Domain Adaptive Control of Nonlinear Rotary Systems Subject to Spatially Periodic Disturbances," Journal of Applied Mathematics, 2012.

[17] L. Zhou, J. She, S. Zhou, and Q. Chen, "Hळ Controller Design for an Observer-Based Modified Repetitive-Control System," International Journal of Engineering Mathematics, 2014.

[18] Y. Wang, R. Wang, X. Xie, and H. Zhang, "Observer-Based Ho Fuzzy Control for Modified Repetitive Control Systems," Neurocomputing, vol. 286, no. 3, pp. 141-149, 2018.

[19] Z. Shao, S. Huang, and Z. Xiang, "Robust Hœ Repetitive Control for a
Class of Linear Stochastic Switched Systems with Time Delay," Circuits, Systems, and Signal Processing, vol. 34, no. 4, pp. 13631377, 2015.

[20] Chi-Ying Lin and Hong-Wu Jheng, "Active Vibration Suppression of a Motor-Driven Piezoelectric Smart Structure Using Adaptive Fuzzy Sliding Mode Control and Repetitive Control," Applied Sciences, vol. 7, no. 3, 2017.

[21] R. Sakthivel, K. Raajananthini, P. Selvaraj, and Y. Ren, "Design and analysis for uncertain repetitive control systems with unknown disturbances," Journal of Dynamic Systems, Measurement, and Control, vol. 140, 2018.

[22] S. Mobayen, "A novel global sliding mode control based on exponential reaching law for a class of underactuated systems with external disturbances," Journal of Computational and Nonlinear Dynamics, vol. 11, no. 2, 2016.

[23] M. Golestani, S. Mobayen, and F. Tchier, "Adaptive finite-time tracking control of uncertain non-linear n-order systems with unmatched uncertainties," IET Control Theory and Applications, vol. 10, no. 14, pp. 1675-1683, 2016.

[24] B. Vaseghi, M. A. Pourmina, and S. Mobayen, "Secure communication in wireless sensor networks based on chaos synchronization using adaptive sliding mode control," Nonlinear Dynamics, 2017.

[25] J. L. Chang, "Dynamic output feedback integral sliding mode control design for uncertain systems," International Journal of Robust and Nonlinear Control, vol. 22, pp. 841-857, 2012.

[26] R. Chuei, Z. Cao, M. Mitrevska, and Z. Man, "Sliding mode based repetitive control for improved reference tracking," Proceedings of 2014 International Conference on Modelling, Identification and Control, Melbourne, Australia, pp. 166-177, 2014.

[27] W. Sun, H. Cai, F. Zhao, Z. Zhong, "Repetitive Control Design of Simulation Turntable Based on Integral," Proceedings of 2012 International Conference on Modelling, Identification and Control, , Wuhan, China, pp. 849-854, 2012.

[28] L. Wu, H. Gao, and C. Wang, "Quasi sliding mode control of differential linear repetitive processes with unknown input disturbance," IEEE Transactions on Industrial Electronics, vol. 58, no. 7, pp. 30593068, 2011.

[29] E. Rogers, K. Galkowski, and D. H. Owens, "Control systems theory and applications for linear repetitive processes," Lecture Notes in Control and Information Sciences, vol. 349, Springer, Germany, 2007.

[30] W. Paszke, K. Galkowski, E. Rogers, and D. H. Owens, "Hळ control of differential linear repetitive processes," IEEE Transactions on Circuits and Systems II, vol. 53, no. 1, pp. 39-44, 2006.

[31] D. S. Yoo and M. J. Chung, "A variable structure control with simple adaptation laws for upper bounds on the norm of the uncertainties," IEEE Transactions on Automatic Control, vol. 37, no. 6, pp. 860-864, 1992.

[32] R. C. Dorf and R. H. Bishop, "Modern control systems," Pearson Prentice Hall, 12th edition, 2011. 